\title{
QUANTIFYING THE EVOLVING MAGNETIC STRUCTURE OF ACTIVE REGIONS
}

\author{
Paul A. Conlon ${ }^{1}$, R.T. James McAteer ${ }^{1,2}$, Peter T. Gallagher ${ }^{1}$, and Linda Fennell ${ }^{1}$ \\ 1 School of Physics, Trinity College Dublin, Dublin 2, Ireland \\ 2 Department of Astronomy, New Mexico State University, NM 88003-8001, USA; mcateer@nmsu.edu \\ Received 2010 April 26; accepted 2010 August 3; published 2010 September 22
}

\begin{abstract}
The topical and controversial issue of parameterizing the magnetic structure of solar active regions has vital implications in the understanding of how these structures form, evolve, produce solar flares, and decay. This interdisciplinary and ill-constrained problem of quantifying complexity is addressed by using a two-dimensional wavelet transform modulus maxima (WTMM) method to study the multifractal properties of active region photospheric magnetic fields. The WTMM method provides an adaptive space-scale partition of a fractal distribution, from which one can extract the multifractal spectra. The use of a novel segmentation procedure allows us to remove the quiet Sun component and reliably study the evolution of active region multifractal parameters. It is shown that prior to the onset of solar flares, the magnetic field undergoes restructuring as Dirac-like features (with a Hölder exponent, $h=-1$ ) coalesce to form step functions (where $h=0$ ). The resulting configuration has a higher concentration of gradients along neutral line features. We propose that when sufficient flux is present in an active region for a period of time, it must be structured with a fractal dimension greater than 1.2 , and a Hölder exponent greater than -0.7 , in order to produce $\mathrm{M}$ - and $\mathrm{X}$-class flares. This result has immediate applications in the study of the underlying physics of active region evolution and space weather forecasting.
\end{abstract}

Key words: magnetic fields - methods: statistical - Sun: flares - turbulence

\section{INTRODUCTION}

Solar active regions are concentrations of intense magnetic field in the atmosphere of the Sun. These highly dynamic, complex structures are rooted in the photosphere, extend into the corona, and are the source of many extreme solar events, such as solar flares and coronal mass ejections (Gallagher et al. 2007). Direct spatially localized measurements of the coronal magnetic field are beyond current instrumentation, due to the tenuous nature of the Sun's atmosphere. However, by studying the evolution of magnetic elements on the photosphere we can gain insight into the physical process that governs the formation and evolution of active regions in the corona.

The building blocks of active regions are flux tubes, which are thought to be formed near the tachocline of the Sun (Miesch 2005). Turbulent sub-surface forces result in a buoyant upward motion that cause the formation of $\Omega$-like loops, which in turn emerge through the photosphere to form active regions. In the low $\beta$ corona, the plasma is restrained to flow along these field lines, thereby creating the loop-like structures evident in extreme ultraviolet images of the Sun. Sunspots are the visible footpoints of these active region loops and appear as collections of positive and negative polarity magnetic flux elements in magnetograms. Flux constantly emerges through the surface of the Sun and interacts with existing active region flux. The system is characterized by a large magnetic Reynolds number, given by $R_{m}=L V / \eta$, where $L, V$, and $\eta$ are the length scale, velocity, and the magnetic diffusivity on the photosphere; for typical photospheric values of these parameters the magnetic Reynolds is $\sim 10^{6}-10^{10}$ (McAteer et al. 2010). Hence, the photosphere is considered a highly turbulent and chaotic environment.

Further evidence of the turbulent nature of active regions is found in the distribution of magnetic flux on the surface of the Sun (Vlahos 2002; Abramenko 2005b; Hewett et al. 2008). The distribution of magnetic flux within active regions follows a power-law distribution, suggesting a self-organized structure and hence numerous nonlinear techniques have been used to study the evolution of active regions (Abramenko 2005a; Abramenko et al. 2008; McAteer et al. 2005a; Conlon et al. 2008; Aschwanden \& Aschwanden 2008). One common method of examining the self similar distribution of active region flux is the fractal dimension. McAteer et al. (2005a) examined $10^{4}$ active region images, related solar flares within a $24 \mathrm{hr}$ window, and found that a fractal dimension of 1.2-1.25 was a minimum requirement for an active region to produce solar flares. Conlon et al. (2008) similarly used a modified box-counting technique to examine the multifractal properties of evolving active regions. They found that as a region developed toward a state favorable to producing solar flares, there was a decrease in both the breadth and height of the multifractal spectrum. An additional method used to examine the fractal and multifractal properties of active regions is the structure function (Abramenko 2005a; Abramenko et al. 2008). A structure function is defined as a statistical moment of the increments of a field and measures its associated intermittences. Abramenko et al. (2008) showed that there is a relation between systematic changes in the ratio of certain powers of the structure function at both the photospheric and chromospheric level. Georgoulis $(2005,2008)$ examined the problems of event prediction with such methods, but supported the use of these methods as analytical tools for the understanding of the fundamental processes involved in the formation and evolution of active regions.

These methods face several problems in dealing with the data presently available; they are threshold-dependent (i.e., results depend on the value chosen to remove the surrounding quiet Sun component) and numerical errors combined with poor spatial resolution result in large errors for large and negative moments (Georgoulis 2005; Conlon et al. 2008). The more stable and reliable wavelet transform modulus maxima (WTMM; Kestener et al. 2010) method is used in this paper in order to overcome these problems, and examine the fractal properties of solar magnetogram data. The WTMM method 
replaces the boxes from the traditional box-counting method with wavelets, which act as fuzzy boxes and are defined for both finite and discrete domains. The observations are described in Section 2. In Section 3, a short description of the WTMM and segmentation method is presented. Analysis of the fractal properties of evolving active regions using this method is shown in Section 4. Our conclusion and future directions are then given in Section 5.

\section{OBSERVATIONS}

The Michelson Doppler Imager (MDI; Scherrer et al. 1995) on board the Solar and Heliospheric Observatory (SOHO; Domingo et al. 1995) images the Sun on a $1024 \times 1024$ pixel CCD camera through a series of increasingly narrow filters. The final elements, a pair of tunable Michelson interferometers, enable MDI to record measurements of the line-of-sight photospheric field with an FWHM bandwidth of $94 \mathrm{~m} \AA$. In this paper, 96 minute cadence magnetograms of the full disc are used, which have a pixel size of $\sim 2^{\prime \prime}$.

A series of magnetograms are analyzed to examine the differences in fractal properties between flaring and non-flaring active regions. Six active regions were analyzed as they evolved and rotated across the solar disk (NOAA AR 10488, 9878, $10763,10942,10954$, and 10956). Given the powerful nature of the WTMM method, and improved accuracy of the segmentation method (see Section 3.2), thresholding was not performed on the data. The analysis of each data set was restricted to periods when the center of each active region was within $\pm 60^{\circ}$ of disk center, in order to decrease the errors associated with projection effects. Magnetograms were corrected assuming a radial field at each point on the solar disk and assuming an equal-area cylindrical projection method (Bugayevskiy \& Snyder 1995; McAteer et al. $2005 \mathrm{~b}$ ) when calculating the basic physical parameters of area and flux.

\section{METHOD}

Traditional box-counting methods (Vlahos 2002) calculate the multifractal parameters of active regions based on the distribution of magnetic flux elements within magnetograms. These methods are prone to errors in the calculation of the multifractal parameters due to image thresholding, resolution, and instrument noise. Wavelet-based methods have been shown to be versatile tools for the study of active region magnetic features (Hewett et al. 2008; Ireland et al. 2008). The WTMM method is particularly versatile and has been used to study X-ray flare emission (McAteer et al. 2007) and aid in the automatic detection of coronal loops (McAteer et al. 2010).

The WTMM method calculates the multifractal parameters based on the distribution of gradients within each image across scale. As such, the multifractal parameters as calculated by the WTMM method are more robust to changes in the resolution and signal-to-noise ratio of magnetogram images. Additionally, the removal of quiet Sun features can be naturally carried out in gradient space as opposed to pixel space, thereby removing the problem of creating singular features in magnetogram images that arises from normal thresholding. This segmentation of quiet Sun, along with the limited resolution of MDI magnetograms, does result in a reduced number of gradients upon which regression can be performed. We used a moving average of five magnetogram images in order to overcome this issue. In this section, the main advantages of using the wavelet transform for performing a multifractal analysis are reviewed.

\subsection{Basics of the 2D Wavelet Tranform Modulus Maxima (WTMM) Method}

A rigorous and complete mathematical description of the WTMM is available in Arneodo et al. (2000) and Kestener et al. (2010). Here, we provide a brief outline to assist the reader in understanding the technique, while retaining the terminology of Arneodo et al. (2000). The three main parts of the WTMM are (1) carry out a wavelet transform, (2) extract the skeleton of lines of modulus maxima, and (3) compute the companion partition functions.

It can be shown that a two-dimensional (2D) continuous wavelet transform, $\mathbf{T}$, of any 2D image, $f(x, y)$, at each location, b, can be obtained as the gradient vector of the image smoothed to various scales, $a$, by a dilating filter, $\phi$,

$$
\begin{aligned}
\mathbf{T}_{\psi}[f](\mathbf{b}, a) & =\nabla\left\{T_{\phi}[f](\mathbf{b}, a)\right\} \\
& =\nabla\left\{\phi_{\mathbf{b}, a} * f\right\},
\end{aligned}
$$

where $\psi$ is the gradient of the filter. For our work, this amounts to smoothing the data by a Gaussian and carrying out a gradient filter in the two image axis, $(x, y)$, to obtain $\mathbf{T}$. The image is then smoothed by a larger Gaussian and the operation is repeated.

At each scale, the WTMM edges are extracted at locations where the wavelet transform modulus $\mathcal{M}_{\psi}[f](\mathbf{b}, a)=$ $\left|\mathbf{T}_{\psi}[f](\mathbf{b}, a)\right|$ is locally maximum in the direction of $\mathbf{T}_{\psi}[f](\mathbf{b}, a)$. These WTMM points lie in connected maxima chains. The wavelet transform skeleton is the set of maxima lines $\mathcal{L}_{\mathbf{x}_{0}}$ defined as the location where the modulus is a local maximum along a maxima chain. These lines contain all the information about the local Hölder regularity $(h)$ properties of the image. Along a maxima line $\mathcal{L}_{\mathbf{x}_{0}}$ that points to $\mathbf{x}_{0}$ in the limit $a \rightarrow 0^{+}$, the wavelet transform modulus behaves as a power law with exponent $h\left(\mathbf{x}_{0}\right)$,

$$
\mathcal{M}_{\psi}[f]\left[\mathcal{L}_{\mathbf{x}_{0}}(a)\right] \sim a^{h\left(\mathbf{x}_{0}\right)} .
$$

The partition function, $\mathcal{Z}$, is calculated from the skeleton by rising the modulus maxima values to some moment, $q$ (where $q$ can take any real value), and summing over all lines,

$$
\mathcal{Z}(q, a)=\sum_{\mathcal{L} \in \mathcal{L}(a)} \mathcal{M}_{\psi}[f](\mathbf{x} \in \mathcal{L}, a)^{q},
$$

from which the scaling exponents, $\tau(q)$, are defined in the limit of small scales $\mathcal{Z}(q, a) \sim a^{\tau(q)}, a \rightarrow 0^{+}$. However, rather than calculating $\tau(q)$, and carrying out a Legendre transform to obtain the multifractal spectrum, Arneodo et al. (2000) suggest computing the companion partition functions directly. The Boltzmann weighting of each skeletal line is defined as $W_{\psi}[f](q, \mathcal{L}, a)=\left(\mathcal{M}_{\psi}[f]\right)^{q} / \mathcal{Z}(q, a)$, the two components of the multifractal spectra, $D(h)$ are

$$
\begin{aligned}
h(q, a)= & \sum_{\mathcal{L} \in \mathcal{L}(a)} \ln \left|\mathcal{M}_{\psi}[f](\mathbf{x}, a)\right| W_{\psi}[f](q, \mathcal{L}, a) \\
& \sim a^{h(q)}, \\
D(q, a)= & \sum_{\mathcal{L} \in \mathcal{L}(a)} W_{\psi}[f](q, \mathcal{L}, a) \ln \left(W_{\psi}[f](q, \mathcal{L}, a)\right) \\
& \sim a^{D(q)},
\end{aligned}
$$

and the linear regime of Equations (4) and (5) are adapted to reflect the physical size of the feature under study. 

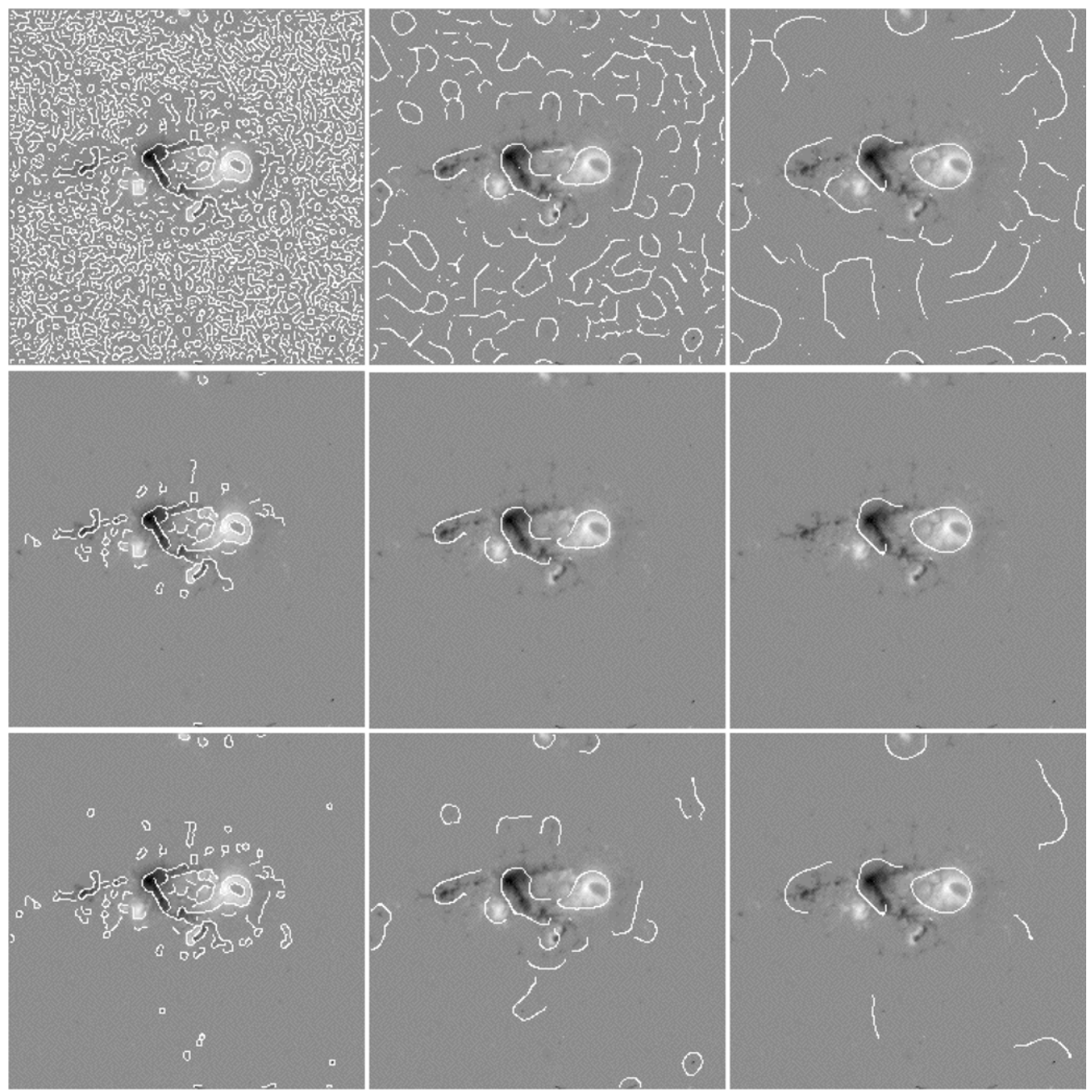

Figure 1. Top: wavelet transform gradients of NOAA 10488 at scales 7, 28, and 56 pixels. Middle: same as top after the segmentation method proposed by Kestener et al. (2010). Bottom: same as top after the segmentation method used in present study (intercept of 40).

\subsection{Segmentation Using the WTMM Method}

The analysis of active region magnetic fields can be misinterpreted due to the contribution of statistical information from the surrounding quiet Sun. It is important to minimize this contribution as much as possible, as the multifractal properties of the quiet Sun are statistically different from that of an active region (Kestener et al. 2010). One method of doing this is the reduction of the image size and therefore minimizing the contribution of quiet Sun in the resulting analysis. However, the dynamic nature of active regions means an adaptive image size is required for larger regions. Kestener et al. (2010) developed a more advanced method for the segmentation of active and quiet Sun features based on thresholds in the wavelet transform modulus space. They propose to discard those skeletal lines which lie in a region of $\mathcal{M}-a$ space (Equation (2)) to allow for the separation of active region and quiet Sun. Figure 1, second panel, illustrates the effect of this segmentation and unfortunately the thresholds remove weaker parts of the active region gradients along with the quiet Sun features. As the goal of our work is the detection of characteristic changes in the structure and complexity of an active region, it is imperative that each active region be studied as a whole. Kestener et al. (2010; Figure 6) show that quiet Sun features have modulus strengths of no more than 40 at the smallest scale; as such, 40 is used as a hard fixed threshold in this study. Figure 1, third panel, shows the remaining skeletons after removing all gradients with a modulus strength below 40 at the smallest scale. This proposed threshold allows for the inclusion of all active region gradients associated with the active region with the suppression of the majority of the quiet Sun gradients.

\section{RESULTS}

The WTMM method discussed above is used to study the evolution of the fractal dimension and its associated Hölder exponent for a number of active regions. These present a single point, $q=0$, on the multifractal $D(h)$ versus $h$ curve.

\subsection{Flaring Regions}

Figure 2 shows NOAA 10488 emerging onto the solar disk on 2003 October 26 and growing rapidly until 2003 October 29. Prior to the emergence of the region only a small number of gradients exceed the thresholds used in segmenting the quiet Sun features, resulting in small and poorly resolved values for $D(h)$. During the emergence period, the Hölder exponent increases as a larger number of skeletal gradients pass the segmentation thresholds. The region produced several flares during two periods of activity. The first $24 \mathrm{hr}$ period of solar flares started around 14:00 UT on 2003 October 27 and contained five C-class flares and an M1.9 flare. The second period started around 18.00 UT on the 2003 October 29, lasted for two days and 


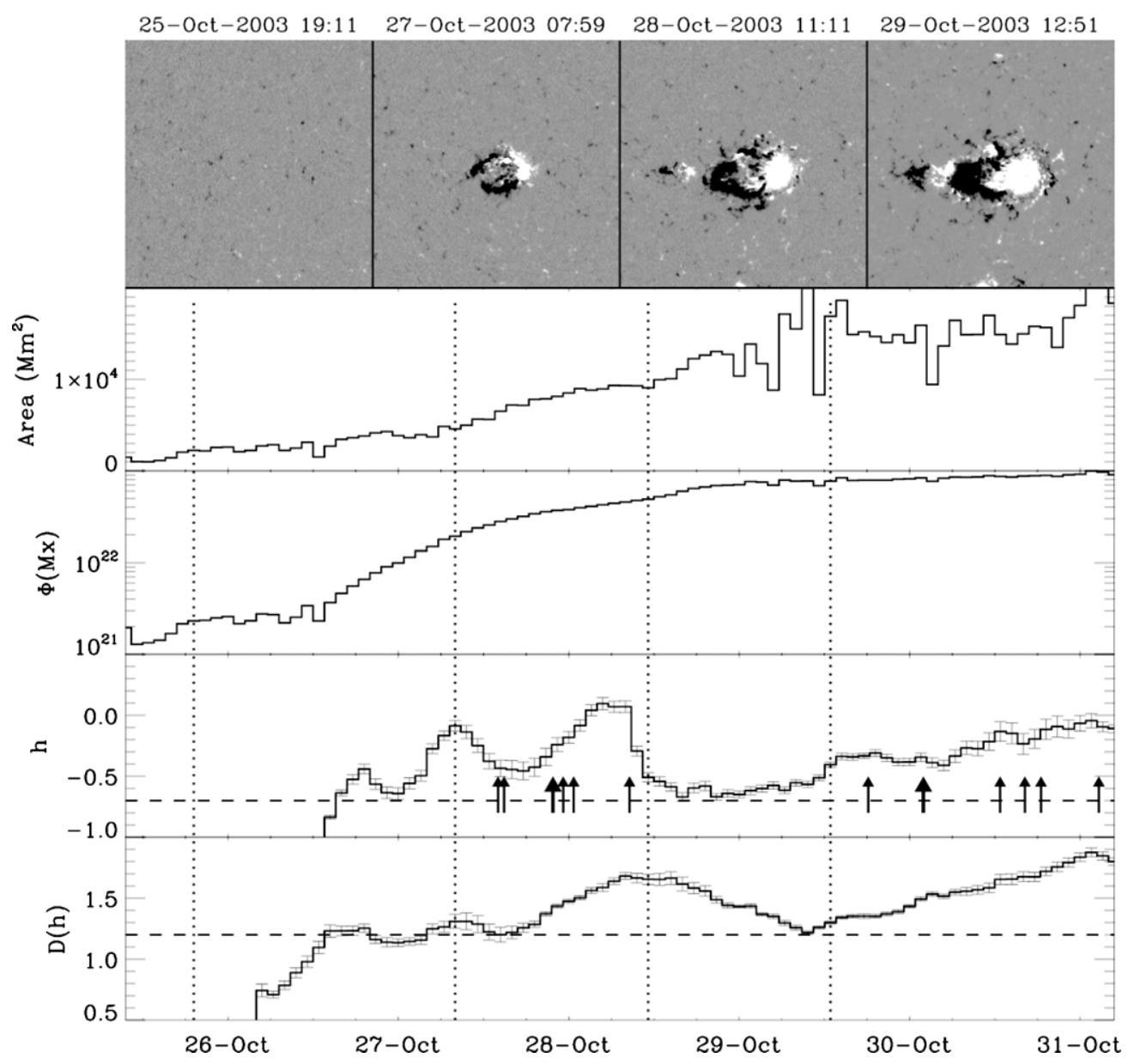

Figure 2. Evolution of NOAA 10488. Top panel: MDI magnetogram images for NOAA 10488 times shown above. Second panel: total area (Mm²). Third panel: total unsigned magnetic flux (Mx). Fourth panel: $h$ for $q=0$. Bottom panel: $D(h)$ for $q=0$. Associated C-class flares are indicated by thin arrows; bolder arrows indicate M-class flares. Vertical dotted lines indicate the time of MDI magnetograms of the top row. Dashed horizontal line in the fourth panel is at $h=-0.7$, a new proposed lower threshold for solar flare production. Dashed horizontal line in the bottom panel highlights a previously proposed threshold of $D(h)=1.2$ for solar flare production (McAteer et al. 2005a). All vertical axes in each of the following Figures 3-7 have the same scaling for direct comparison.

resulted in eight $\mathrm{C}$-class flares and two M-class flares. Flaring periods are associated with a fractal dimension above 1.2 and a Hölder exponent greater than that calculated for quiet-Sun features, $h>-0.7$ (Kestener et al. 2010). This suggests a reorganization of the underlying magnetic field. It should be noted that $h=-1$ corresponds to Dirac white noise, $h=0$ a step function, and $h=1$ a smooth differential function. As the Hölder exponent increases, Dirac-like gradients are replaced by more structured step-function-like gradients. The formation of large structured gradients in the region allows for the storage and release of larger amounts of free magnetic energy.

Figure 3 shows NOAA 10763 rotating into view on 2005 May 15 after which both the area and magnetic flux remained relatively stable. Around 2005 May 15 17:00 UT the region entered a period of solar flare productivity, releasing twelve C-class and four M-class flares. During this flaring period the fractal dimension and Hölder exponent were around or above the proposed thresholds for flaring. Some small changes in the Hölder exponent seem to be associated with these flares. Prior to some flares there is an increase in the Hölder exponent, with a subsequent dip following the flare. This dip in the Hölder exponent can be understood as the loss of coherent structure among the magnetic gradients present in the region or a reduction in the amount of magnetic free energy in the region. The subsequent rise in the Hölder exponent and clustering of the gradients in the region into a coherent structure accompanies the release of an M-class flare.

Figure 4 shows NOAA 9878, which rotated onto the solar disk on 2002 March 22 as a fully developed, large, stable region. The region produced four C-class flares over a $36 \mathrm{hr}$ period beginning around 10:00 UT on 2002 March 25. Similarly to NOAA 10763 and NOAA 10488, this period of solar flares is associated with a fractal dimension greater than 1.2 and an increase in the Hölder exponent to above -0.7 . After this period of solar flares, the Hölder exponent and fractal dimension decreased to quiet Sun levels, accompanying the decrease in magnetic free energy available in the region.

It is well established that solar flares are associated with active regions that are large in area and magnetic field strength. However, these examples show that solar flares are also associated with an increased fractal dimension $(D(h)>1.2)$ and Hölder exponent $(h>-0.7)$. In the following section, we describe active regions which do not produce solar flares, in order to corroborate these results.

\subsection{Non-flaring Regions}

Figure 5 shows NOAA 10954 as it emerged on 2007 April 28 and rotated across the disk before decaying on 2007 May 5. This was a small region with a maximum area of $\sim 4 \times 10^{3} \mathrm{Mm}^{2}$ and maximum flux of $\sim 7 \times 10^{21} \mathrm{Mx}$. 


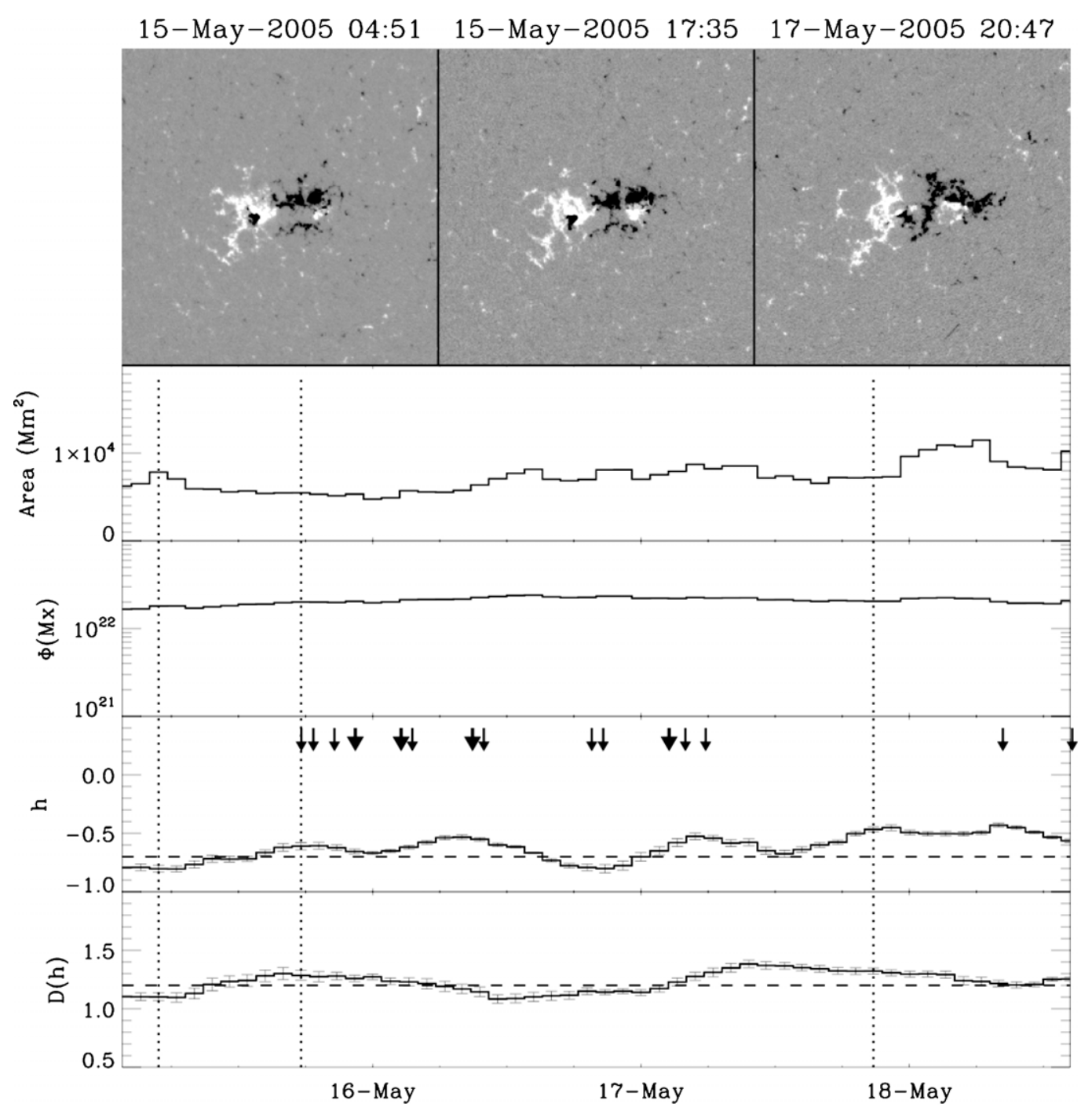

Figure 3. Evolution of NOAA 10763. Top panel: MDI magnetogram images for NOAA 10763 at the times shown. Second panel: total area (Mm²). Third panel: total unsigned magnetic flux (Mx). Fourth panel: $h$ for $q=0$. Bottom panel: $D(h)$ for $q=0$. Associated C-class flares are indicated by thin arrows; bolder arrows indicate M-class flares. Vertical dotted lines indicate the time of MDI magnetograms and horizontal lines are proposed thresholds for solar flare production.

The region maintained a high Hölder exponent and fractal dimension, indicating a possibility of solar flares, but did not produce any flares. This demonstrates that fractal properties alone are not reliable for the prediction of flares and other physical properties, such as area and total magnetic field strength, must be taken into account.

Figure 6 shows NOAA 10942, a small region of maximum area of around $5 \times 10^{3} \mathrm{Mm}^{2}$ and a field strength of $9 \times 10^{21} \mathrm{Mx}$, which emerged on 2007 February 17. The Hölder exponent remains above the proposed threshold of $h>-0.7$. However, the fractal dimension only briefly rises above the proposed threshold of $D(h)>1.2$. This suggests that although the region was structured in a manner favorable to flaring (large Hölder exponent), the region was not large enough (in area or flux) or concentrated enough (as indicated by the low fractal dimension) in order to store sufficient magnetic energy for solar flares.

Figure 7 shows NOAA 10956, which rotated around the east limb on 2007 May 15. Apart from a C-class flare while on the limb, the region produced no further flares. The magnetic flux reached a peak of $1 \times 10^{22} \mathrm{Mx}$ and the area peaked at $3 \times 10^{4} \mathrm{Mm}^{2}$. Given the large amount of magnetic flux present in the region, the occurrence of solar flares was likely. However, as the Hölder exponent oscillates around the proposed threshold, and the fractal dimension remains below, the region is never structured in a manner favorable to producing solar flares.

These results show that even in active regions seemingly containing sufficient flux, the magnetic field must be structured in a sufficiently complex configuration in order to produce flares. Similarly, a complex configuration is insufficient by itself (without a large flux), to produce flares. In the three active regions studied here, one of either the magnetic flux, fractal dimension, or Hölder exponent is too small and so no solar flares occur.

\section{CONCLUSIONS AND FUTURE WORK}

The WTMM method is shown to be a stable and accurate mathematical tool for the analysis of magnetic fields on the solar disk. The WTMM method allows for the calculation of the magnetic field fractal dimension and Hölder exponent based on the distribution of gradients within the magnetic structure across scale space. The segmentation of quiet Sun magnetic 


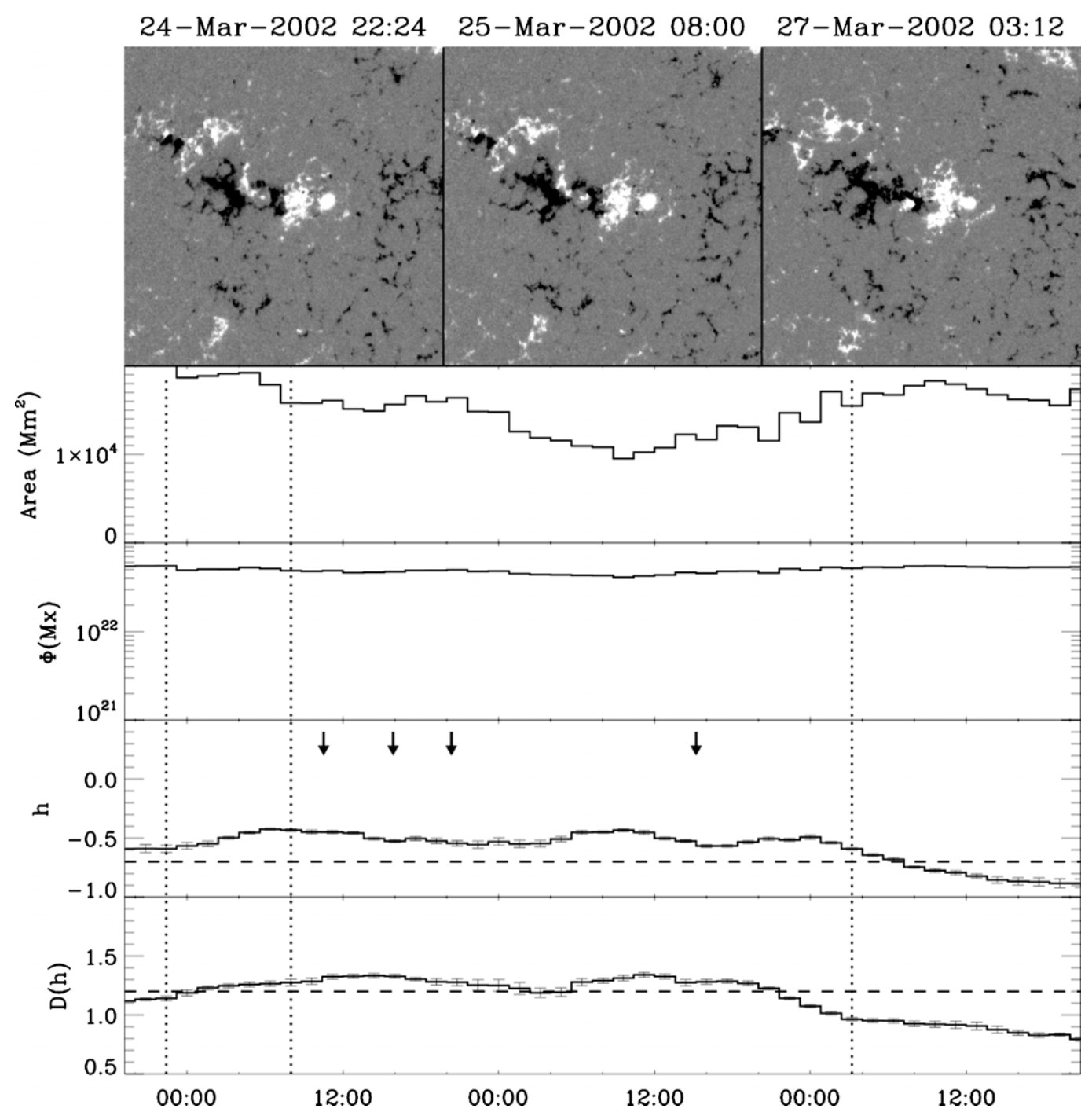

Figure 4. Evolution of NOAA 9878. Top panel: MDI magnetogram image for NOAA 9878 at the times shown. Second panel: total area (Mm²). Third panel: total unsigned magnetic flux (Mx). Fourth panel: $h$ for $q=0$. Bottom panel: $D(h)$ for $q=0$. Associated C-class flares are indicated by thin arrows; bolder arrows indicate M-class flares. Vertical dotted lines indicate the time of MDI magnetograms and horizontal lines are proposed thresholds for solar flare production

information in gradient space allows for the sole study of each active region without the introduction of artifacts associated with thresholding. Table 1 outlines our results and shows a strong link between size, complexity, and solar flares. Similar to the results of McAteer et al. (2005a), a lower threshold in of $D(h)>1.2$ is found to be a necessary but insufficient condition for the occurrence for solar flares. We propose a further constraint of a Hölder exponent of no less than -0.7 as an indicator of the future occurrence of solar flares. The Hölder exponent detects the restructuring of active regions from a field representing Diraclike gradients toward one closer to step-function-like gradients. Thus, if a region is sufficiently large in both area and flux, and has a sufficiently high fractal dimension and Hölder exponent, the possibility of the region producing solar flares is greatly increased.

It is clear that the fractal dimension and Hölder exponent offer great insight into the structure of active regions, however a cautionary note is necessary as this small sample size restricts us to only qualitative statements regarding solar flare probabilities. As suggested by McAteer et al. (2010), the likely nonlinear nature of the driver of flares (i.e., reconnection) combined with the unknown exact link between the location of the measurements (i.e., the photosphere) and the location of the event (i.e., the corona) may make reliable solar flare predictions an almost impossible task. However, the results presented here do confirm the ability of multiscale methods to detect characteristic changes in magnetic elements on the photosphere of the Sun (Hewett et al. 2008; Abramenko 2005b). In particular, those characteristics such as changes in the flatness of structure functions, the dimensional and contribution diversity of the multifractal spectrum, and the fractal dimension (Abramenko 2005a; Conlon et al. 2008; McAteer et al. 2005a) are associated with an enhanced probability of producing flares. The work presented here is an improvement on each of these methods and allows for a more detailed analysis than was previously possible of the processes involved in the evolution of active regions.

There are several remaining weak points in this method. While the best effort has been made to remove the quiet Sun component, this problem remains open to interpretation. Our chosen value of 40 , used to threshold the wavelet transform modulus gradients at the smallest scale, was chosen so as to maximize the contribution of gradients associated with active 


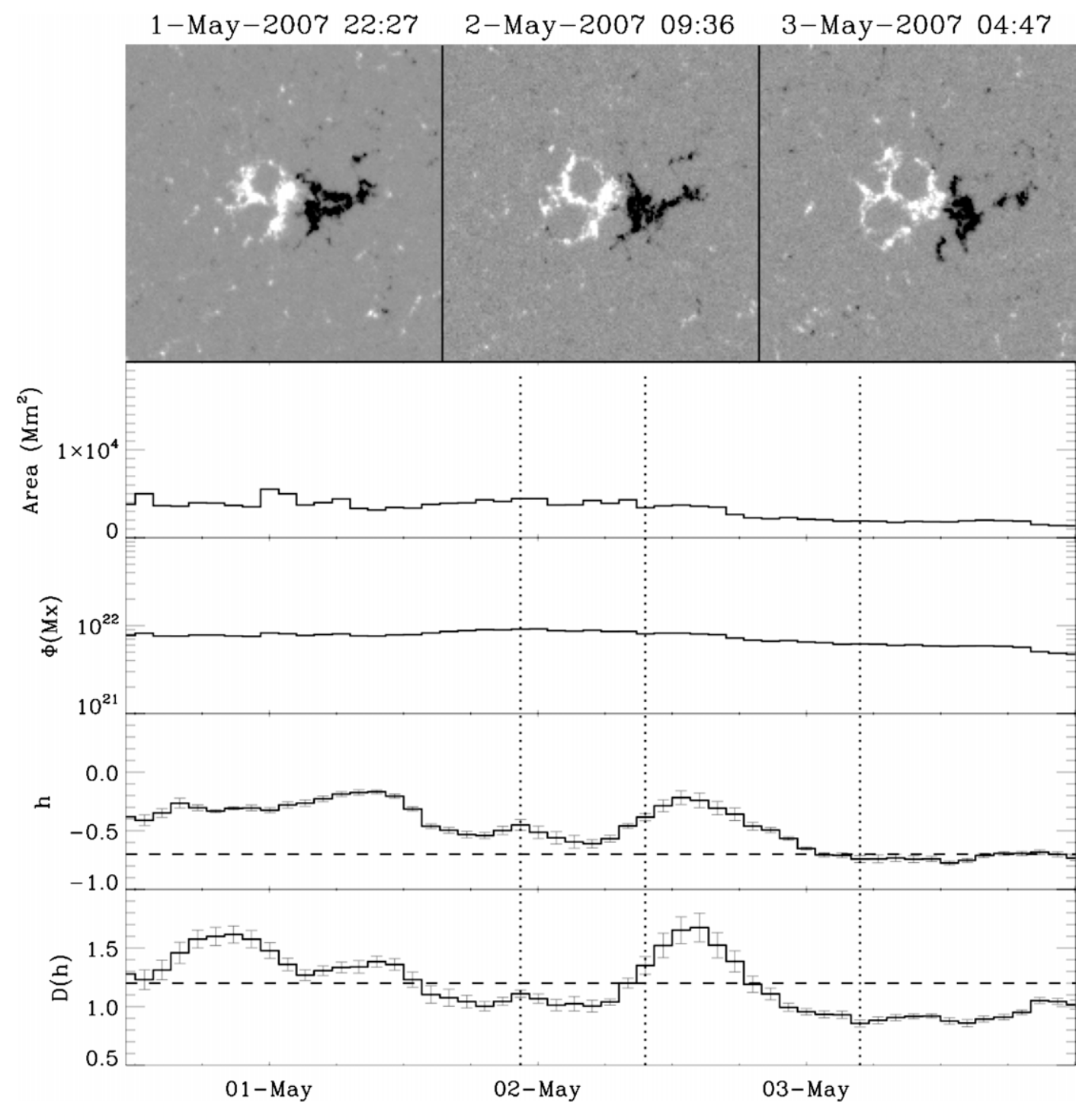

Figure 5. Evolution of NOAA 10954. Top panel: MDI magnetogram image for NOAA 10954 at the times shown. Second panel: total area $\left(\mathrm{Mm}^{2}\right)$. Third panel: total unsigned magnetic flux (Mx). Fourth panel: $h$ for $q=0$. Bottom panel: $D(h)$ for $q=0$. Vertical dotted lines indicate the time of MDI magnetograms and horizontal lines are proposed thresholds for solar flare production

regions and minimize the number associated with quiet Sun features. Active regions of all sizes contain a large number of low strength gradients. As such, the incorporation of the slope threshold in modulus space may reduce the number of these low strength gradients in the calculations. Given the ranges of scales present in active regions these results are also limited by the use of an arbitrary and fixed regression range. While great care has been taken to select the most appropriate range of scales for each image, an automated regression technique is required to improve on these results. Furthermore, it is unclear how changes in the resolution of magnetogram images will affect the different methods used to calculate the multifractal parameters of active region. As the WTMM method calculates these parameters based on the distribution of gradients within the image, it should be more robust to changes in resolution (as compared to the traditional box-counting method). The enhanced detail offered by an increase in resolution would increase the number of visible gradients within the images and hence remove the need for a running average of magnetograms.
These results represent only one data point in the multifractal spectrum, the so-called fractal dimension or $q=0$. The WTMM method allows for the calculation and analysis of the full multifractal spectrum. This is akin to performing traditional emission-line spectroscopy, studying the amplitude and wavelength of the peak, but ignoring the width and asymmetry between the wings. As such, a future investigation into the behavior of the whole spectrum will provide additional information into the changing structure of active region magnetic fields. Given the results of Conlon et al. (2008), changes in the full multifractal spectrum may provide a clearer distinction between flaring and non-flaring regions. Taken together with other physical parameters, the multifractal spectrum may make it possible to detect the restructuring of active region magnetic fields prior to flaring. Further work is needed on a larger set of active regions (McAteer et al. 2005b; Higgins et al. 2010) to fully access the capability of the WTMM method in order to detect the conditions favorable for flaring and further constrain any thresholds that may or may not exist. 


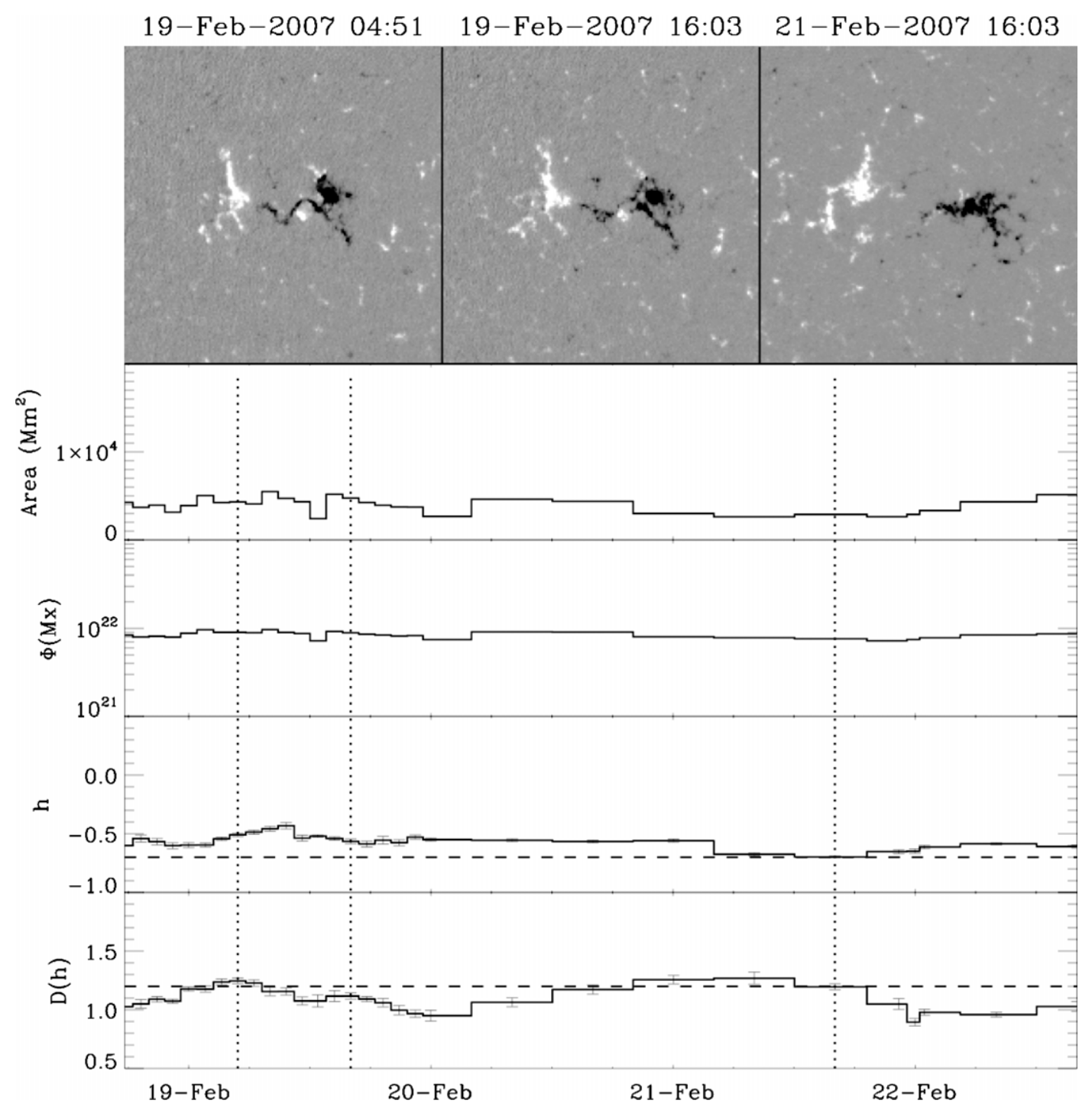

Figure 6. Evolution of NOAA 10942. Top panel: MDI magnetogram image for NOAA 10942 at the times shown. Second panel: total area $\left(\mathrm{Mm}^{2}\right)$. Third panel: total unsigned magnetic flux (Mx). Fourth panel: $h$ for $q=0$. Bottom panel: $D(h)$ for $q=0$. Vertical dotted lines indicate the time of MDI magnetograms and horizontal lines are proposed thresholds for solar flare production

Table 1

Summary of Results for the Six Active Regions Studied

\begin{tabular}{lccccc}
\hline \hline $\begin{array}{c}\text { NOAA } \\
\text { AR }\end{array}$ & $\begin{array}{c}\text { Total Area } \\
\left(\mathrm{Mm}^{2}\right)\end{array}$ & $\begin{array}{c}\text { Magnetic Flux } \\
\left(10^{22} \mathrm{Mx}\right)\end{array}$ & Fractal Dimension & Hölder Exponent & Flares \\
\hline 10488 & 22921 & 9.95 & 1.88 & 0.10 & $13 \mathrm{C} \& 3 \mathrm{M}$ \\
10763 & 11482 & 2.40 & 1.38 & -0.43 & $12 \mathrm{C} \& 4 \mathrm{M}$ \\
9878 & 23415 & 5.48 & 1.34 & -0.42 & $4 \mathrm{C}$ \\
10954 & $\mathrm{X}$ & $\mathrm{X}$ & $\mathrm{X}$ & -0.58 & $\mathrm{X}$ \\
10942 & $\mathrm{X}$ & $\mathrm{X}$ & $\mathrm{X}$ & -0.56 & $\mathrm{X}$ \\
10956 & 13522 & 2.26 & $\mathrm{X}$ & $\mathrm{X}$ & $\mathrm{X}$ \\
\hline
\end{tabular}

Note. Maximum values for the total area, magnetic flux, Fractal Dimension, and Hölder exponent are presented when they exceed thresholds for an extended period of time.

The authors acknowledge the helpful advice and support of Dr. Kestener, Dr. Khalil, and Dr. Arneodo, along with Dr. Georgoulis and Dr. Abramenko for several fruitful discussions on this topic, and the referee and editor for suggesting several useful changes to the manuscript. The au- thors thank the $\mathrm{SOHO} / \mathrm{MDI}$ consortia for their data. SOHO is a joint project by ESA and NASA. This research was supported by a grant from the "Ulysses-Ireland-France Exchange Scheme" operated by the Royal Irish Academy and the Ministère des Affaires Etrangères. P.A.C. is an IRCSET Government of 


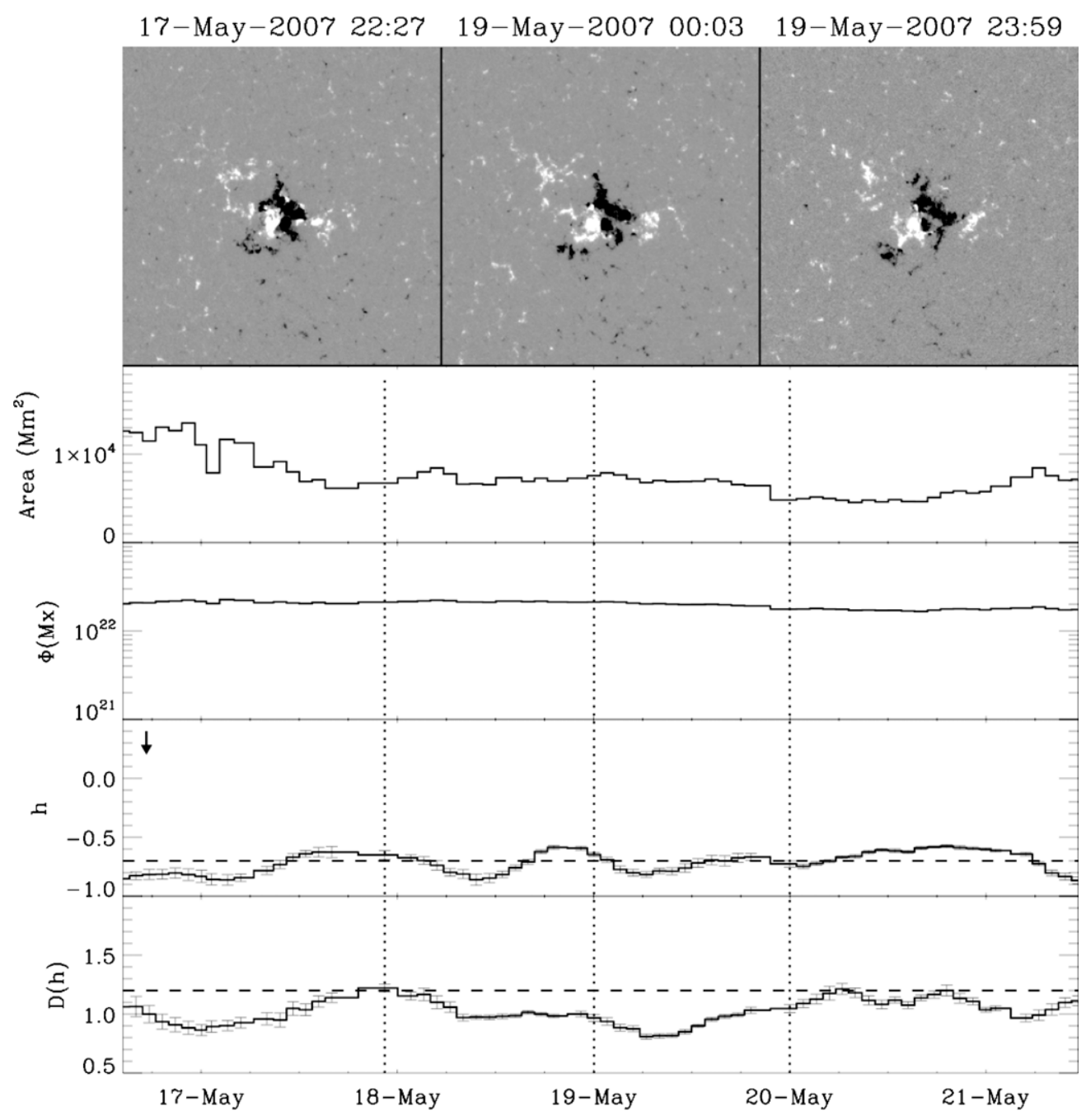

Figure 7. Evolution of NOAA 10956. Top panel: MDI magnetogram image for NOAA 10956 on at the times shown. Second panel: total area $\left(\mathrm{Mm}^{2}\right)$. Third panel: total unsigned magnetic flux (Mx). Fourth panel: $h$ for $q=0$. Bottom panel: $D(h)$ for $q=0$. Vertical dotted lines indicate the time of MDI magnetograms, the associated C-class flare is indicated by the thin arrow, and horizontal lines are proposed thresholds for solar flare production.

Ireland Scholar. R.T.J.M. is PI of a Marie Curie Fellowship under FP6.

\section{REFERENCES}

Abramenko, V., Yurchyshyn, V., \& Wang, H. 2008, ApJ, 681, 1669

Abramenko, V. I. 2005a, Sol. Phys., 228, 29

Abramenko, V. I. 2005b, ApJ, 629, 1141

Arneodo, A., Decoster, N., \& Roux, S. G. 2000, Eur. Phys. J. B, 15, 567

Aschwanden, M. J., \& Aschwanden, P. D. 2008, ApJ, 674, 530

Bugayevskiy, L. M., \& Snyder, J. P. 1995, Map Projections: A Reference Manual (London: Taylor and Francis)

Conlon, P. A., Gallagher, P. T., McAteer, R. T. J., Ireland, J., Young, C. A., Kestener, P., Hewett, R. J., \& Maguire, K. 2008, Sol. Phys., 248, 297

Domingo, V., Fleck, B., \& Poland, A. I. 1995, Sol. Phys., 162, 1

Gallagher, P. T., McAteer, R. T. J., Young, C. A., Ireland, J., Hewett, R. J., \& Conlon, P. 2007, in Space Weather: Research Towards Applications in Europe 2nd European Space Weather Week (ESWW2), ed. J. Lilensten (Astrophysics and Space Science Library, Vol. 344 (Dordrecht: Springer)), 15

Georgoulis, M. K. 2005, Sol. Phys., 228, 5

Georgoulis, M. K. 2008, Geophys. Res. Lett., 35, 6
Hewett, R. J., Gallagher, P. T., McAteer, R. T. J., Young, C. A., Ireland, J., Conlon, P. A., \& Maguire, K. 2008, Sol. Phys., 248, 311

Higgins, P., Bloomfield, D., McAteer, R. J., \& Gallagher, P. T. 2010, Adv. Space Res., doi:10.1016/j.asr.2010.06.024

Ireland, J., Young, C. A., McAteer, R. T. J., Whelan, C., Hewett, R. J., \& Gallagher, P. T. 2008, Sol. Phys., 252, 121

Kestener, P., Conlon, P., Khalil, A., Fennell, L., McAteer, R., Gallagher, P., \& Arneodo, A. 2010, ApJ, 717, 995

McAteer, R. J., Gallagher, P. T., \& Conlon, P. A. 2010, Adv. Space Res., 45, 1067

McAteer, R. T. J., Gallagher, P. T., \& Ireland, J. 2005a, ApJ, 631, 628

McAteer, R. T. J., Gallagher, P. T., Ireland, J., \& Young, C. A. 2005b, Sol. Phys., 228, 55

McAteer, R. T. J., Kestener, P., Arneodo, A., \& Khalil, A. 2010, Sol. Phys., 262, 387

McAteer, R. T. J., Young, C. A., Ireland, J., \& Gallagher, P. T. 2007, ApJ, 662, 691

Miesch, M. S. 2005, Living Rev. Solar Phys., 2, 1

Scherrer, P. H., et al. 1995, Sol. Phys., 162, 129

Vlahos, L. 2002, in SOLMAG 2002: Proc. Magnetic Coupling of the Solar Atmosphere Euroconference, ed. H. Sawaya-Lacoste (ESA Special Publication, Vol. 505; Noordwijk: ESA), 105 